\title{
Lithium-ion battery modeling using CC-CV and impedance spectroscopy characterizations
}

\author{
Tillmann Stübler ${ }^{1} \cdot$ Amine Lahyani $^{2,3}$ (D) Amen Allah Zayoud ${ }^{2}$
}

Received: 24 October 2019 / Accepted: 2 April 2020 / Published online: 5 April 2020

(c) Springer Nature Switzerland AG 2020

\begin{abstract}
The evolution in battery technology is the key to developing the most efficient Electric Vehicles and winning the challenge for the future E-mobility. As it is difficult to describe battery behavior, we seek in this study to determine an accurate circuit model of the battery that can be used in simulation software. Different tests were performed on Panasonic model NCR18650B lithium batteries in order to find an accurate model that characterizes their behavior in four essential criteria: state of charge, internal resistance, differential capacitance and efficiency. The novelty of this work is the implementation of a technical approach that considers DC and AC model characteristics with their respective experimental approaches, which leads to achieving reliable model performance in both stationary and transient load conditions. The validation of this model using the results from constant current experiments was performed by impedance spectroscopy. It enables the validation of equivalent circuit models for the battery that reproduce faithfully its dynamic response at one particular state of charge.
\end{abstract}

Keywords Lithium ion battery $\cdot$ Modeling $\cdot$ RC circuits $\cdot$ Constant current $\cdot$ Constant voltage $\cdot$ Impedance spectroscopy

$\begin{array}{ll}\text { Nomenclature } \\ \text { SoC } & \text { State of charge } \\ \text { CC } & \text { Constant current } \\ \text { CV } & \text { Constant voltage } \\ \text { EIS } & \text { Electrochemical impedance spectroscopy } \\ \text { EV } & \text { Electrical vehicle } \\ \text { BMS } & \text { Battery management system }\end{array}$

\section{Introduction}

Due to the aggravation of environment problems caused by an increased number of Internal Combustion Engine Vehicles (ICEV), there is an increased interest in the research and development of batteries used in Electric and Hybrid Vehicles (EV and HEV). These vehicles are currently seen as a potential alternative for the future of road transportation, taking into consideration the interest in reducing greenhouse gas emissions, as well as air and sound pollution.

According to the International Energy Agency, the transportation sector represents one of the main determinant factors of climatic changes with $23 \%$ of the greenhouse gases in the atmosphere coming from this sector, making it the second largest contributor after the industrial sector [1]. European legislation encourages the industry to develop Electric Vehicles as a replacement to old generation cars. In this context, all big car manufacturers are rushing to win a large part of a fast-growing market, which might put an end to a traditional industry and create new value-added materials, products, services, and potential business models. The bold aim of Germany's "National Electro-Mobility Development Plan" is to have 1 million EV on the roads of Germany by 2020 [2].

Amine Lahyani, amlahyani@yahoo.fr; Tillmann Stübler, tillmann.stuebler@akka.eu; Amen Allah Zayoud, zayoud.amen@outlook.com | 1 AKKA Germany, Posener Str. 1, 71065 Sindelfingen, Germany. ${ }^{2}$ Laboratoire des Systèmes Electriques, Ecole Nationale d'Ingénieurs de Tunis, Université de Tunis el Manar, LR11ES15, 1002 Tunis, Tunisia. ${ }^{3}$ Institut National des Sciences Appliquées et de Technologie, University of Carthage, 1080 Tunis, Tunisia. 
Likewise, there is a tight link between the need for energy storage in $\mathrm{EV}$ and in modern electricity networks since more generating capacity uses renewable energy sources which are intrinsically intermittent. In [3] the authors show that storage systems such as batteries are used in grid-connected electric vehicles to solve the financial support problem of supportive policies in wind energy resources. They highlighted the impact of these vehicles, when integrated with wind resources, on increasing the capacity factor of wind resources. Papers $[4,5]$ study the displayed performance to obtain an optimal design of a hybrid renewable energy scheme (Wind/PV/Battery). The authors minimize the "Total Life Cycle Cost of the scheme subject to relevant limitations by modifying three decision variables, namely, the swept area of the wind turbines, the PV surface area, and the number of batteries. In [6] optimization techniques are presented for a PV/WG/diesel system. They showed the optimal number of system components by which the system can satisfy the demanded load. Simulation results demonstrated that adding a battery bank to the conventional PV/WG/diesel/system gives better cost-effectiveness than without battery. In reference [7], the authors demonstrated that Grid Independent Hybrid Renewable Energy Systems (GIHRES) using battery storage are more cost-effective than those with only hydrogen energy storage. They also stated that the hybridization of solar power, battery, and Reverse Osmosis Desalination at various maximum loss of power supply probability is the most cost-effective energy system.

Focusing on Electric Vehicles, the battery represents the key element as it costs up to $40 \%$ of the total value. It is therefore considered the heart of the electric mobility development process. The key to conquering the EV market is to develop the most efficient and affordable batteries capable of covering longer driving distances. For that reason, automotive industries are putting more effort and resources into battery R\&D. As of now, lithium-ion batteries appear to be the most suitable power storage systems for electric vehicles thanks to their high energy density and increased power per mass battery unit.

Moreover, many researches have focused on the specific type of Lithium battery [2], leading to the development of batteries with reduced weight and dimensions at competitive prices. The improvement of batteries characteristics requires performing various electrochemical tests on them. This allows fully characterization of these batteries and subsequently building representative models that describe their dynamic behavior.

Knowing the importance of this process in battery development, AKKA Germany $[8,9]$ defined the main purpose of this research work as preparing and performing different tests on a specific type of battery (Panasonic NCR18650B used in TESLA Cars), using a lithium-ion battery test bench developed inside the company. The second task of the study is to analyze the retrieved data in order to extract a representative model that characterizes these batteries behavior versus time, temperature and electrical waveforms.

The remainder of the paper is as follows. Section 2 presents a description of the used working environment, tools and methods. Sections 3 and 4 present a detailed description of the tests performed on the batteries and give an analysis of the obtained measurements in order to establish an accurate battery model. Section 5 describes the methodology implemented to establish the battery model. The paper concludes with both the quantitative and qualitative outcomes of this research work as well as its perspectives.

\section{Presentation of the project environment}

In order to study and model the behavior and the physical characteristics of the battery, we used a test-bench designed specifically for this purpose. It consists of five identical modules that operate independently from each other. The cells are placed inside a climatic chamber during experiments.

\subsection{Test bench architecture}

Every battery test bench unit includes three main parts: BMS, Control/Supervising Unit and Li-ion cells.

The BMS is any electronic system that manages a rechargeable battery: it ensures the charging and discharging function, monitors the state of the battery, protects the battery by preventing it from working outside its safe operating area, and measures and reports the battery data. In this project, the BMS is an electronic module equipped with electrical excitations for the charging and discharging process and was completely designed inside the company.

The BMS operates under the command of a single board computer Raspberry Pi 3 model B. The test bench operator controls the raspberry pi via SSH connection. The tests were performed on Panasonic NCR18650B battery cells, which have a rated capacity of $3100 \mathrm{mAh}$ and a maximum charging voltage of $4.2 \mathrm{~V}$. Panasonic also specifies a maximum charging current of $2 \mathrm{~A}$ per cell. We use the 4-wire measurement technique to measure cell voltage, thus eliminating the effect of lead resistance [10].

For measuring cell temperature, PT1000 sensors are glued on the cell surface. The 3-wire technique is used to eliminate lead resistance. For every cell, two sensors are placed at different positions on the cell for redundancy and cross-validation purposes. 
All analog measurements are done with ADS1262 analog-to-digital converters. Their internal programmable analog front-end includes several features that are used to improve measurement accuracy and reduce circuit complexity. For cell current measurement, programmable gain stages are used depending on the expected current magnitudes.

For temperature measurement, the internal matched current sources are used to realize a measurement bridge and the ADC chopper mode and programmable gain amplifiers are used to achieve good measurement performance.

\subsection{CC-CV operating mode}

The test bench offers two operating modes for signal generation:

\subsubsection{Constant voltage}

The measurement module behaves as a voltage source. Charging and discharging currents will depend on the difference between the open circuit voltage and the cell voltage applied. The test bench supports an open-loop mode and a closed-loop mode.

In open-loop mode, the shunt resistor for current sensing as well as the lead wires to the battery sum up to a total resistance of the voltage source. In closed-loop mode, an integrating controller adjusts the voltage source to eliminate any voltage drop that occurs in the shunt resistor or lead wires. The controller was implemented in software and relies on the ADC readings, so the response time depends on the sample rate. This mode is less suitable for dynamic or transient tests.

\subsubsection{Constant current}

The measurement module behaves like a current source, again employing a software control loop. With any of those three modes, we can generate several waveforms like sine, triangular or windowed (band-limited) sine, which is used for impedance spectroscopy.

\section{Constant current tests}

By convention, SoC is defined as the amount of charge stored in the battery, relative to a well-defined reference state. The reference state is the fully-charged $(U=4.2 \mathrm{~V})$ and stationary battery. With this convention, SoC is not relative to the battery's total capacity, thus it was unnecessary to measure it. The cycle starts by discharging the battery until reaching $2.8 \mathrm{~V}$. The battery is then stabilized for $1 \mathrm{~h}$ at $2.8 \mathrm{~V}$ before charging it with the same constant current until reaching $4.2 \mathrm{~V}$ again. Before each CC cycle, the battery is stabilized at $4.2 \mathrm{~V}$ for several hours. The measurement modules record voltage and current at a rate of $2.5 \mathrm{~Hz}$.

The same test protocol was carried at temperatures $10{ }^{\circ} \mathrm{C}, 20^{\circ} \mathrm{C}, 30^{\circ} \mathrm{C}$ and $40{ }^{\circ} \mathrm{C}$ respectively. At $20^{\circ} \mathrm{C}$, the experiment was performed with current ranges of $20 \mathrm{~mA}, 50 \mathrm{~mA}, 100 \mathrm{~mA}, 200 \mathrm{~mA}, 500 \mathrm{~mA}$ and $1000 \mathrm{~mA}$. For the other temperatures, the most time-consuming low-current tests were not performed due to time constraints (Fig. 1).

\subsection{State of charge}

Neglecting self-discharge, the amount of charge stored in the cell is calculated according to the Coulomb counting method as follows:

$Q(t)=Q_{0}+\int_{t_{0}}^{t} I(t) d t$

First, we calculate charge content $Q(t)$ as the integral of cell current $I(t)$, where, by convention, $Q=0$ applies to the fully-charged stationary cell and $I$ is positive when charging and negative when discharging. Then, we create an equally spaced vector of 1000 charge values $Q_{i}$ from $Q_{\min }$ through $Q_{\max }$ and perform a piecewise linear least-squares fit of $I(Q)$ and $U(Q)$ to reduce the amount of data. This is done separately for the discharging and charging measurements. We obtain $Q_{i}, U_{d, i}, U_{c, i} I_{d, i} I_{c, i}$ at 1000 points $[11,12]$.

Decreasing the data to 1000 will not induce any loss in usable information. The results for various constant current cycles, all performed at $20^{\circ} \mathrm{C}$, are shown in Fig. 2 .

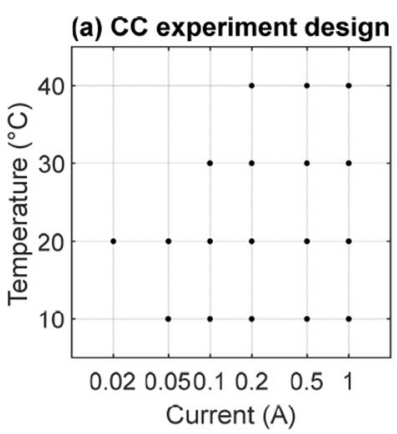

(b) IS experiment design

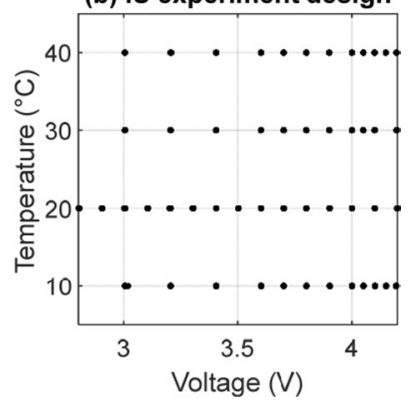

Fig. 1 Experiment design 


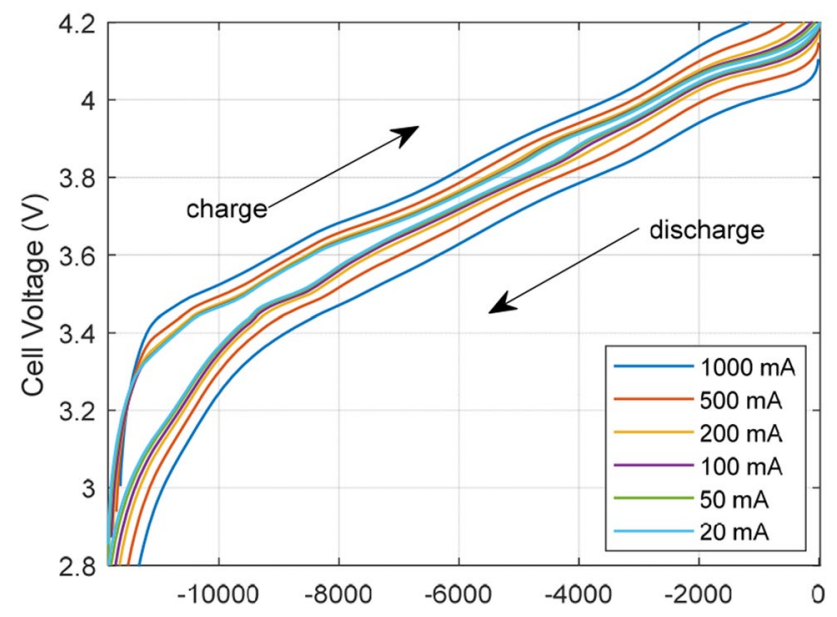

Fig. 2 Voltage versus $Q$ for different currents at $20^{\circ} \mathrm{C}$

\subsection{Internal resistance}

Internal resistance is closely related to internal dissipation and battery efficiency, thus it is of great interest. The internal resistance in this section is determined by constant current tests. Other methods used for its determination, like Impedance spectroscopy, will be discussed in detail in the next section. Applying Ohm's law, an equivalent resistance called "effective resistance" could be determined by dividing the voltage difference by the change in current [13-15]. Contrary to the common approach of using pulse response experiments for internal resistance estimation, we used the difference in cell voltage between CC charging and discharging at any particular SoC to obtain what we call quasi-stationary resistance.

So we obtain:

$R_{k}=\frac{U_{c, k}-U_{d, k}}{I_{c, k}-I_{d, k}}$

The internal resistance is often put in series with the open circuit voltage of the battery. It generates an internal voltage decrease. If there is no current in the battery, the voltage drop due to the internal resistance is equal to zero. Thus, the voltage at the terminals is equal to the open circuit voltage. If a specific current is extracted or delivered to the battery (positive or negative for charge/ discharge phases), the resistance of the external load becomes in series with the battery resistance $R_{k}$.

A battery is a concrete complex circuit since it can behave as resistive, capacitive or inductive impedance. Furthermore, equivalent circuit models can not precisely describe the battery's behavior under all conditions. Therefore, when using the term «internal resistance» for batteries, we need to precisely describe the method and conditions used in its estimation [16].

Figure 3 displays different internal resistance variation for different currents. The results confirm the fact that ohm's law does not apply to the internal resistance provided by the experimental method that was described.

\subsection{Differential capacitance}

From the same raw data, we can calculate the cell's differential capacitance as a function of equilibrium voltage, as shown in Fig. 4 for $I=20$ mA. It was calculated according to Eq. 3 as follows:

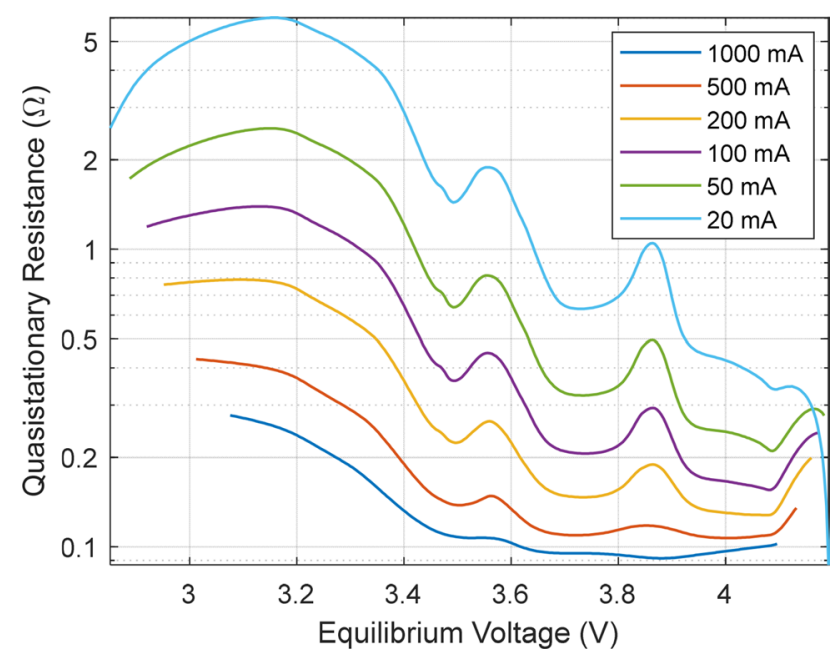

Fig. 3 Quasi-stationary resistance of the battery at $20^{\circ} \mathrm{C}$

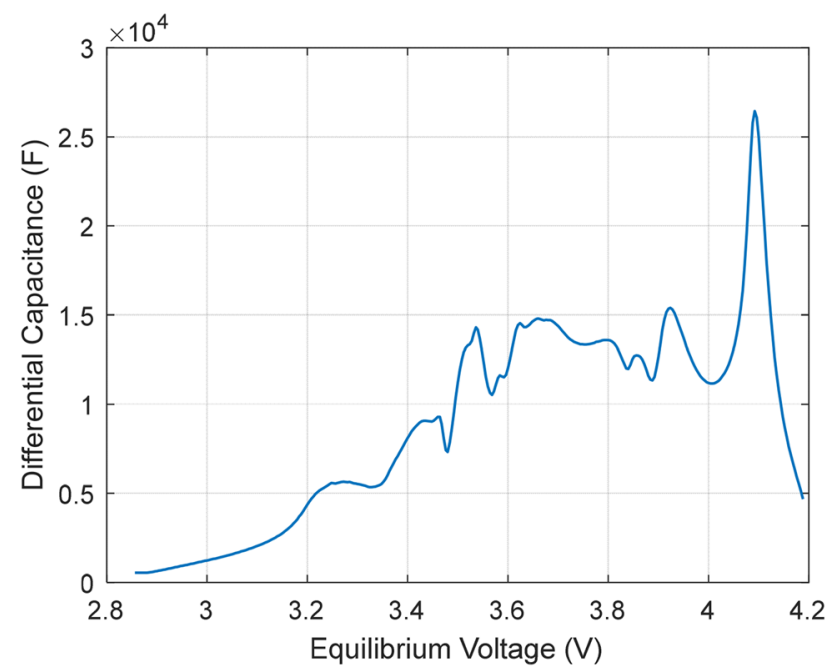

Fig. 4 Differential capacitance of the battery at $20^{\circ} \mathrm{C}$ 
$C_{k}=\frac{Q_{k+1}-Q_{k}}{U_{k+1}-U_{k}}$

where $U_{k}$ is averaged for charge and discharge.

The differential capacitance varies for different currents. However, for low currents, the curves converge to a certain limit. At high currents, we obtain different results since calculation is based on the assumption of quasi-stationary operation, which is not valid at high currents.

\subsection{Battery efficiency}

The energetic efficiency is a key parameter for the overall performance of a battery. We consider studying the energetic efficiency by dividing the discharging energy by the charging energy of the battery as given by Eq. 4 :

$\eta(I)=\frac{\left|W_{d}\right|}{\left|W_{c}\right|}=-\frac{\int_{\text {Disch arge }} U_{d} I_{d} d t}{\int_{\text {Charge }} U_{c} l_{c} d t}$

Figure 5 confirms that the efficiency increases with lower constant current values.

If we consider the battery as an RC series circuit as for the internal resistance, then we have to expect the efficiency to come near $100 \%$ for very low currents. However, this is not the case. This finding indicates that linear equivalent circuit models can not exactly reproduce the battery's behavior.

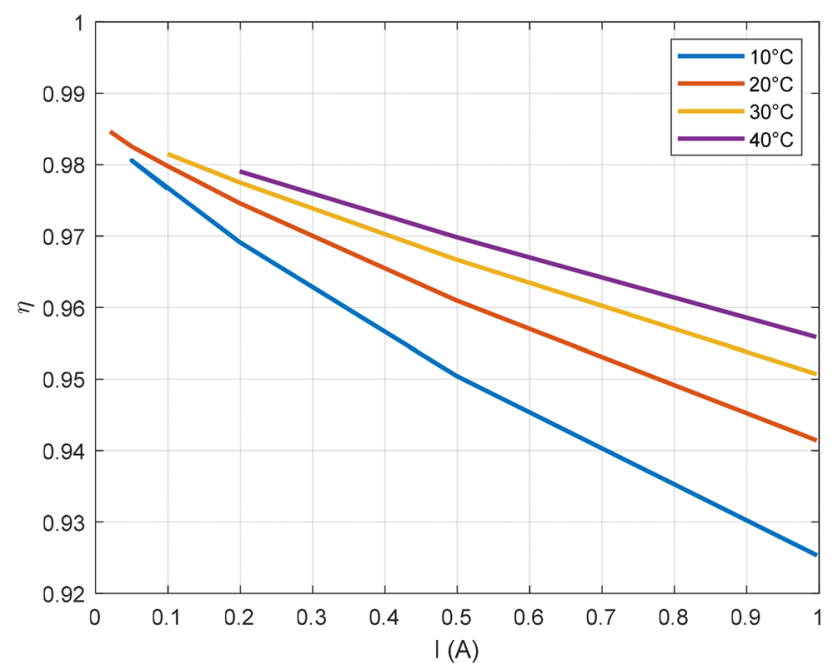

Fig. 5 Cycle efficiency of the battery at different temperatures

\section{Impedance spectroscopy tests}

The most common and standard approach used for impedance spectroscopy is done by applying a single-frequency voltage or current to the interface and measuring the phase shift and amplitude, or real and imaginary parts, of the resulting current at that frequency using Fast Fourier Transform (FFT) analysis of the response. The impedance is measured as a function of frequency. The advantages of this approach are the availability of these instruments and the ease of their use, as well as the fact that the experimentalist can achieve an adequate signal-to-noise ratio in any frequency range [17].

The impedance spectroscopy consists of small amplitude voltage excitation for a specific frequency and for a specific time where the generated signal is sine modulated with a Gaussian Pulse window function. The impedance spectroscopy test is time-consuming, but it provides a good insight into the battery's behavior and allows the estimation of the ECM parameters. In other words, it is a very efficient approach for modeling the battery.

The impedance is calculated according to Eq. 5:

$\underline{Z}(i \omega)=|\underline{Z}(\omega)| e^{j \phi(\omega)}=\frac{\int e^{-j \omega t} U(t) d t}{\int e^{-j \omega t} /(t) d t}$

Before applying the previous formula, some preprocessing is applied to the raw data such as offset elimination, resampling and windowing.

The impedance spectrograms cover 25 frequencies equally spaced in log-scale between $316 \mu \mathrm{Hz}$ and $5.6 \mathrm{~Hz}$. The same test protocol was performed at temperatures $10{ }^{\circ} \mathrm{C}, 20^{\circ} \mathrm{C}, 30^{\circ} \mathrm{C}$ and $40^{\circ} \mathrm{C}$, for each temperature at 15 different SoCs corresponding to cell voltages $2.8 \mathrm{~V}, 2.9 \mathrm{~V}$, until $4.2 \mathrm{~V}$.

Then we plot the imaginary part of the impedance versus the real part. Figure 6 illustrates the impedance spectrum for three voltage levels at $20^{\circ} \mathrm{C}$.

\section{Battery modeling results and discussion}

In order to create a model for the battery, we start by adopting an equivalent circuit model. Then we deduce the values of the circuit elements via optimization of the mean-squared difference between the modeled and the measured impedances.

The equivalent circuit model is composed of a capacitor (models the differential capacitance of the battery), a resistor (models the high-frequency resistance) and a number of RC circuits (Fig. 7). For each impedance, we have one complex equation to solve, given by: 


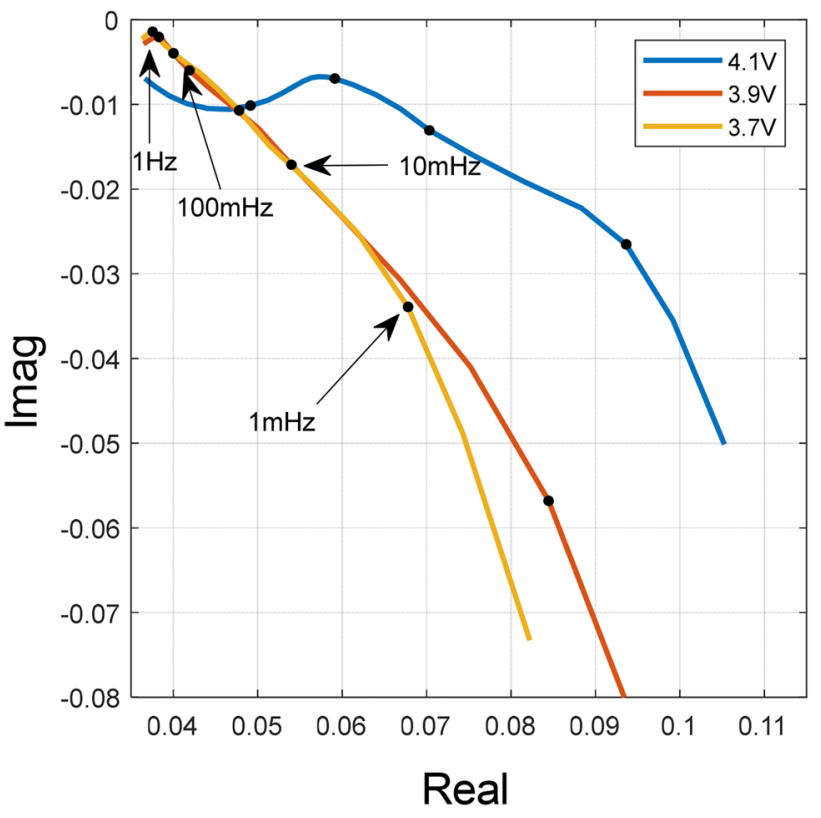

Fig. 6 Impedance spectrums for voltages $3.7 \mathrm{~V}$ to $4.1 \mathrm{~V}$ at $20^{\circ} \mathrm{C}$

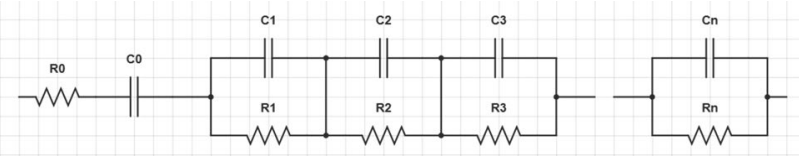

Fig. 7 Battery voigt circuit model

$\underline{Z}(j \omega)=R_{0}+\frac{1}{j C_{0} \omega}+\sum_{k=1}^{N} \frac{R_{k}}{1+j \omega \tau_{k}}$

with $\tau_{k}=R_{k} C_{k}$. We separate the complex equations into their real and imaginary parts and solve the whole system using a trust-region algorithm (Isqnonlin function in MATLAB).

For that, we need to implement the latter equation and its Jacobi matrix with respect to the unknown parameters. Equations are scaled with the reciprocal absolute value of measured impedance to achieve approximately equal sensitivity to measurement errors for all frequencies. Heuristics are used to obtain suitable initial parameters.

The optimization converges quickly after about 10 iterations. The number of RC circuits must be chosen carefully. Too many RC circuits worsen model performance due to overfitting. For many applications, 2-4 RC circuits are sufficient.

To validate the models obtained, we compare the model's internal resistance and differential capacitance to the results obtained from constant current experiments. The quasi-stationary internal resistance in the ECM is as follows:

$R_{D C}=R_{0}+\sum_{k=1}^{N} R_{k}$

For this model, four RC circuits are used and IS values $R_{/ S}$ and $C_{1 S}$ in the range [4.1-3.6 V] are estimated with a step of $0.1 \mathrm{~V}$. The range was chosen based on its relevance for lithium batteries.

The Constant Current and Impedance Spectroscopy tests are two different approaches that could be used for internal resistance determination. While the first one defines "large signal resistance", the second defines "small signal resistance". These two definitions could be connected with each other using system theory for non-linear systems.

Table 1 contains the model parameters for the 3RC model at $20^{\circ} \mathrm{C}$ and three different SoCs.

In Fig. 8, we plot the internal resistance and differential capacitance of the obtained model with those obtained from the constant current tests at current value equal to $1 \mathrm{~A}$. We can see only a rough correlation of both methods. Again, this indicates an insufficiency of the equivalent circuit model, even though the reproduced impedance spectrum is very close to the measured one for a wide range of frequencies covering $316 \mu \mathrm{Hz}$ until $5.6 \mathrm{~Hz}$ (Fig. 9).

Increasing the number of RC circuits $(n>4)$ does not give better congruency with the $C C$ results, but increases the risk of over-fitting due to the large number of unknown parameters.

\section{Conclusion and perspectives}

In order to describe the complex electric behavior of lithium ion batteries with "black box" approaches, the electro-chemical system is regarded as a specific two-terminal device. Two different perspectives are

Table 1 Obtained model parameters

\begin{tabular}{lllll}
\hline Voltage & Unit & $3.7 \mathrm{~V}$ & $3.9 \mathrm{~V}$ & $4.1 \mathrm{~V}$ \\
\hline$R_{\text {tot }}$ & $\Omega$ & 0.077 & 0.099 & 0.101 \\
$C_{0}$ & $\mathrm{~F}$ & 7323 & 4144 & 10,709 \\
$R_{0}$ & $\Omega$ & 0.0345 & 0.0349 & 0.0348 \\
$R_{1}$ & $\Omega$ & 0.0037 & 0.0045 & 0.0212 \\
$R_{2}$ & $\Omega$ & 0.0095 & 0.0151 & 0.0129 \\
$R_{3}$ & $\Omega$ & 0.0292 & 0.0445 & 0.0317 \\
$\tau_{1}$ & $\mathrm{~S}$ & 0.0372 & 0.0520 & 0.0372 \\
$\tau_{2}$ & $\mathrm{~S}$ & 4.2240 & 4.6757 & 3.3125 \\
$\tau_{3}$ & $\mathrm{~S}$ & 86.3517 & 90.2201 & 64.2093 \\
\hline
\end{tabular}



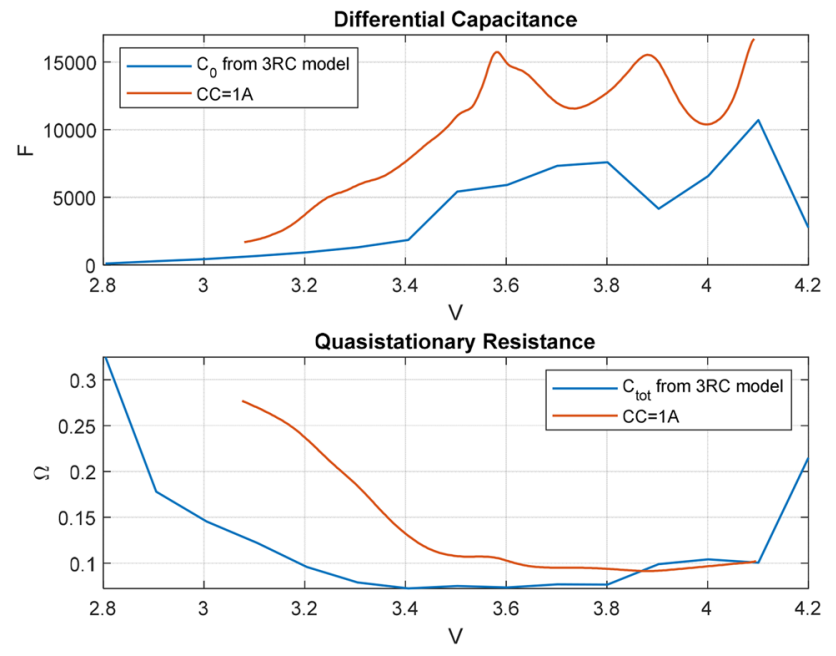

Fig. 8 Comparison between internal resistance and differential capacitance with IS and CC methods

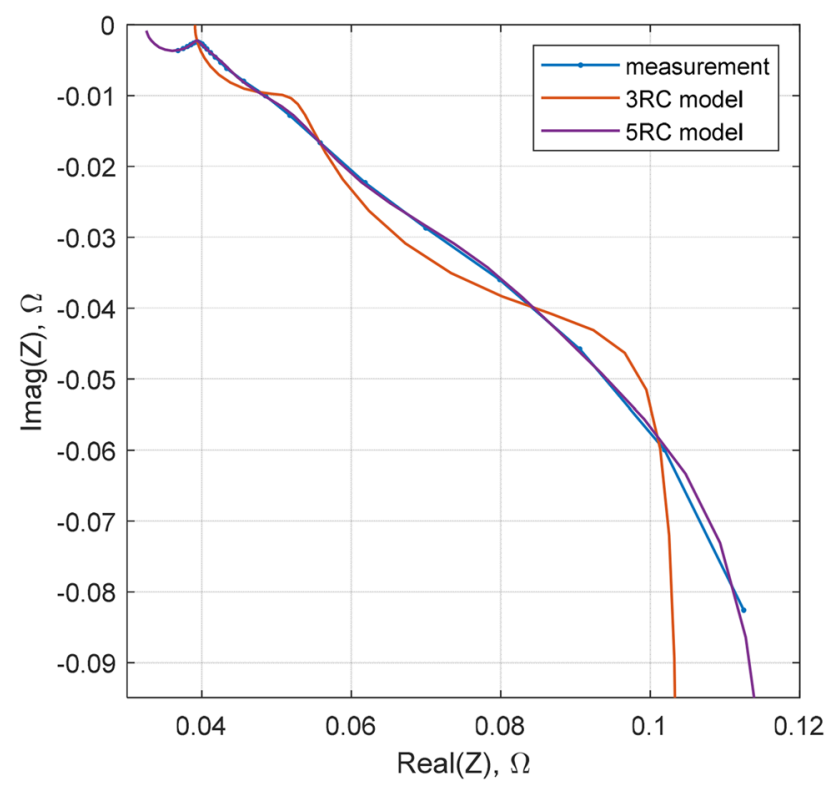

Fig. 9 Reproduced Impedance spectra at 4.0 V using 3 and 5 RC circuits

commonly used to characterize certain features of that device: quasi-stationary (or DC characteristics) and AC characteristics. Those perspectives correspond to two complementary experimental approaches: Constant Current experiments and Impedance Spectroscopy. On the other hand, each of them corresponds to a simplified abstraction of black box battery models. Concerning the parameter identification of equivalent circuit models, common approaches aim to identify both DC and AC characteristics with a single experimental approach like the step response method.
In this paper, we presented a different method which aims to specifically consider DC and AC model characteristics with their respective experimental approaches, in the hope of achieving reliable model performance in both stationary and transient load conditions.

In the experimental part of this study, an extensive characterization of the cell type NCR18650B (used in Tesla cars) was performed. The result obtained is a wide band impedance spectrogram for each point in a temperature-SoC grid, and one constant current charging/ discharging cycle for each point at a temperature-cell current grid. Performing these tests at different temperatures provided an insight of the temperature influence on the dissipative and dynamic behavior of the battery.

The modeling part of the study relied upon those experimental results. A certain equivalent circuit model was chosen. Model parameters were estimated for every temperature-SoC point separately by least squares model fitting applied to the measured impedance spectra. The result is that the found models have very close dynamic behavior to the considered cell type at that particular temperature and SoC. Those models were then used to derive their quasi-stationary characteristics, which were compared to the measured DC characteristics from the constant current experiments. The results show rough correlation of the modeled and measured DC properties. This indicates that the proposed method could be a useful approach for robust model parameter identification and validation.

Acknowledgments The authors would like to thank "Akka Technologies" and "PARTNERSHIPS FOR ENHANCED ENGAGEMENT IN RESEARCH (PEER)" for their technical and financial support.

Funding This study was funded by AKKA GmbH \& Co. KGaA Posener Str. 1 - 71065 Sindelfingen - Germany.

\section{Compliance with ethical standards}

Conflict of interest The authors attest that no conflict of interest exists in this work.

\section{References}

1. Iclodean C, Varga B, Burnete N, Cimerdean D, Jurchiș B (2017) Comparison of different battery types for electric vehicles. IOP Conf Ser 252:12058

2. Di Bitonto S, Trost R (2015) Electromobility in Germany: vision 2020 and beyond. Germany Trade and Invest, Berlin

3. Motie S, Keynia F, Ranjbar MR, Maleki A (2016) Generation expansion planning by considering energy-efficiency programs in a competitive environment. Int J Electr Power Energy Syst 80:109-118 
4. Zhang W, Maleki A, Rosen MA (2019) A heuristic-based approach for optimizing a small independent solar and wind hybrid power scheme incorporating load forecasting. J Clean Prod 241:117920

5. Zhang W, Maleki A, Rosen MA, Liu J (2018) Optimization with a simulated annealing algorithm of a hybrid system for renewable energy including battery and hydrogen storage. Energy 163(15):191-207

6. Maleki A, Pourfayaz F (2015) Sizing of stand-alone photovoltaic/ wind/diesel system with battery and fuel cell storage devices by harmony search algorithm. J Energy Storage 2:30-42

7. Maleki A (2018) Design and optimization of autonomous solarwind-reverse osmosis desalination systems coupling battery and hydrogen energy storage by an improved bee algorithm. Desalination 435:221-234

8. AKKA Technologies website (2019) https://www.akka-techn ologies.com Accessed 02 Sept 2019

9. wiki/Akka_Technologies (2019) website https://fr.wikipedia.org/ wiki/Akka_Technologies Accessed 04 Sept 2019

10. Mamaradlo JS (2019) Four-wire sensing can make or break your measurements. https://www.electronicdesign.com/blog/fourwire-sensing-can-make-or-break-your-measurements Accessed 14 Sept 2019

11. Hawley G (2017) Understanding Tesla's lithium ion batteries. https://evannex.com/blogs/news/understanding-teslas-lithi um-ion-batteries, Accessed 11 Sept 2019
12. Baccouche I, Jemmali S, Mlayah A, Manai B, Essoukri Ben Amara $\mathrm{N}$ (2018) Implementation of an improved coulomb-counting algorithm based on a piecewise SOC-OCV relationship for SOC estimation of Li-ion battery. Int J Renew Energy Res 8:10654

13. Ratnakumar BV, Smart MC, Whitcanack LD, Ewell RD (2006) The impedance characteristics of Mars exploration rover Li-ion batteries. J Power Sources 159(2):1428-1439

14. Copetti JB, Chenlo F (1994) Lead/acid batteries for photovoltaic applications. Test results and modeling. J Power Sources 47(1-2):109-118

15. Barsoukov E, Ross Macdonald J (2005) Impedance spectroscopy: theory, experiment and applications, 2nd edn. Wiley, Hoboken

16. Fitting EIS Data - Diffusion Elements - Warburg, 24 July 2014, https://www.consultrsr.net/resources/eis/diffusion.htm Accessed 13 Sept 2019

17. Huet $F$ (1998) A review of impedance measurements for determination of the state-of-charge or state-of-health of secondary batteries. J Power Sources 70:59-69

Publisher's Note Springer Nature remains neutral with regard to jurisdictional claims in published maps and institutional affiliations. 